\section{Notification List}

Correspondence

Jean Euzéby

email address can be found at www.bacterio.net

\section{Notification that new names and new combinations have appeared in volume 57 , part 4 , of the IJSEM}

This listing of names published in a previous issue of the IJSEM is provided as a service to bacteriology to assist in the recognition of new names and new combinations. This procedure was proposed by the Judicial Commission [Minute 11(ii), Int J Syst Bacterio/ 41 (1991), p. 185]. The names given herein are listed according to the Rules of priority (i.e. page number and order of valid publication of names in the original articles). Taxonomic opinions included in this List (i.e. the creation of synonyms or the emendation of circumscriptions) cannot be considered as validly published nor, in any other way, approved by the International Committee on Systematics of Prokaryotes and its Judicial Commission.

\begin{tabular}{|c|c|c|}
\hline Name/author(s): & Proposed as: & Page no. \\
\hline Nocardia ninae Laurent et al. 2007 & sp. nov. & 664 \\
\hline Bibersteinia Blackall et al. 2007 & gen. nov. & 672 \\
\hline $\begin{array}{l}\text { Bibersteinia trehalosi (Sneath and Stevens 1990) Blackall et al. } 2007 \text { (basonym Pasteurella } \\
\text { trehalosi Sneath and Stevens 1990) }\end{array}$ & comb. nov. & 673 \\
\hline Halomonas gomseomensis Kim et al. 2007 & sp. nov. & 678 \\
\hline Halomonas janggokensis Kim et al. 2007 & sp. nov. & 678 \\
\hline Halomonas salaria Kim et al. 2007 & sp. nov. & 680 \\
\hline Halomonas denitrificans Kim et al. 2007 & sp. nov. & 680 \\
\hline Leucobacter iarius Somvanshi et al. 2007 & sp. nov. & 685 \\
\hline Aeromicrobium panaciterrae Cui et al. 2007 & sp. nov. & 690 \\
\hline Sulfitobacter litoralis Park et al. 2007 & sp. nov. & 694 \\
\hline Trabulsiella odontotermitis Chou et al. 2007 & sp. nov. & 699 \\
\hline Prolixibacter Holmes et al. 2007 & gen. nov. & 705 \\
\hline Prolixibacter bellariivorans Holmes et al. 2007 & sp. nov. & 705 \\
\hline Lactobacillus farraginis Endo and Okada 2007 & sp. nov. & 711 \\
\hline Lactobacillus parafarraginis Endo and Okada 2007 & sp. nov. & 711 \\
\hline Microlunatus ginsengisoli Cui et al. 2007 & sp. nov. & 715 \\
\hline Haloferax larsenii Xu et al. 2007 & sp. nov. & 718 \\
\hline Actinoplanes couchii Kämpfer et al. 2007 & sp. nov. & 722 \\
\hline Moryella Carlier et al. 2007 & gen. nov. & 726 \\
\hline Moryella indoligenes Carlier et al. 2007 & sp. nov. & 728 \\
\hline Granulicoccus Maszenan et al. 2007 & gen. nov. & 733 \\
\hline Granulicoccus phenolivorans Maszenan et al. 2007 & sp. nov. & 733 \\
\hline Natronorubrum sulfidifaciens Cui et al. 2007 & sp. nov. & 739 \\
\hline Luteimonas composti Young et al. 2007 & sp. nov. & 742 \\
\hline Lactobacillus secaliphilus Ehrmann et al. 2007 & sp. nov. & 748 \\
\hline Vagococcus elongatus Lawson et al. 2007 & sp. nov. & 753 \\
\hline Pseudochrobactrum kiredjianiae Kämpfer et al. 2007 & sp. nov. & 759 \\
\hline Echinicola vietnamensis Nedashkovskaya et al. 2007 & sp. nov. & 763 \\
\hline Tamlana Lee 2007 & gen. nov. & 766 \\
\hline Tamlana crocina Lee 2007 & sp. nov. & 767 \\
\hline Alkalibacillus silvisoli Usami et al. 2007 & sp. nov. & 773 \\
\hline Nocardioides marinus Choi et al. 2007 & sp. nov. & 778 \\
\hline Haloplanus Elevi Bardavid et al. 2007 & gen. nov. & 782 \\
\hline Haloplanus natans Elevi Bardavid et al. 2007 & sp. nov. & 783 \\
\hline
\end{tabular}


cont.

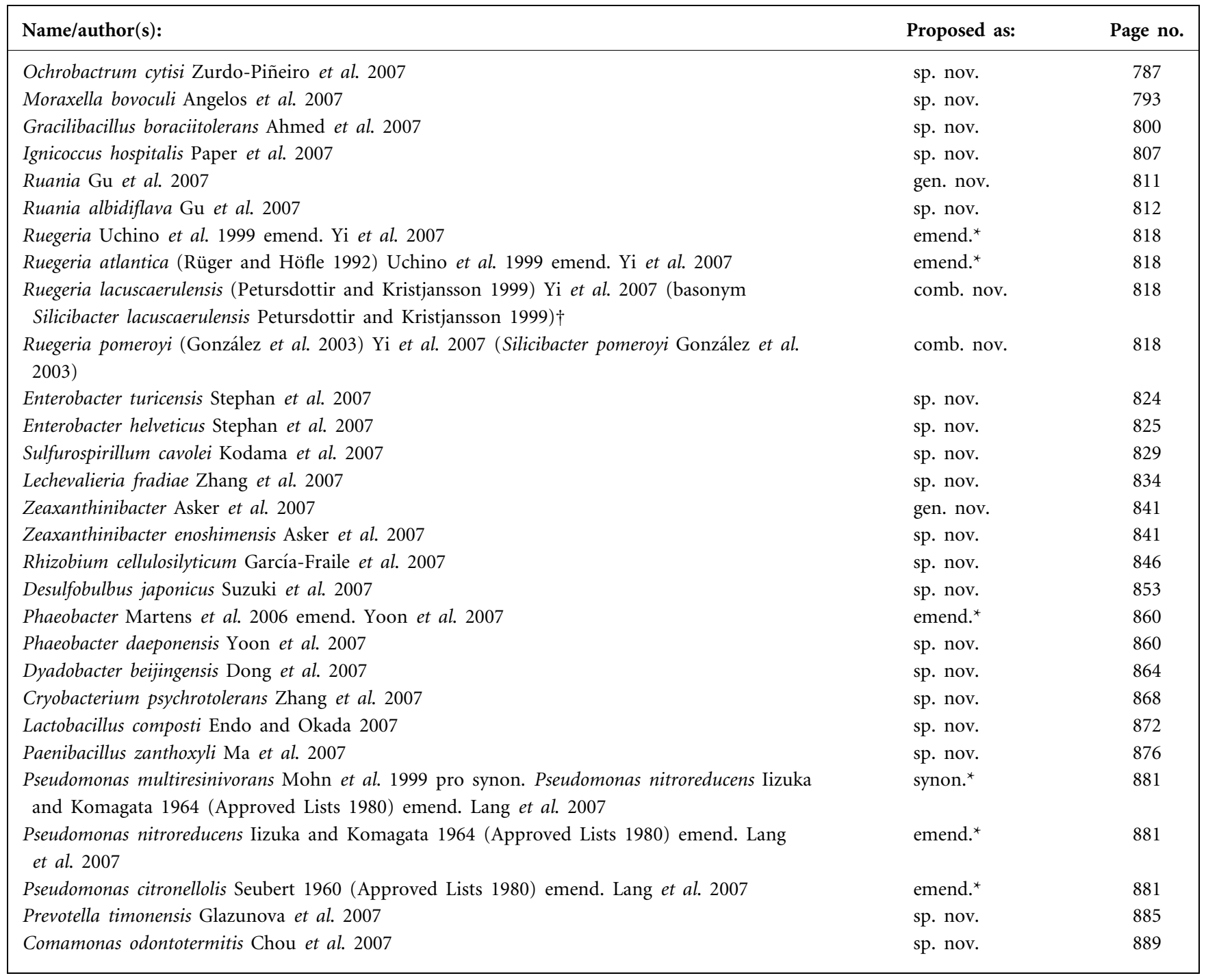

${ }^{\star}$ Taxonomic opinion.

$\dagger$ †i et al. (2007) propose to transfer Silicibacter lacuscaerulensis Petursdottir and Kristjansson 1999 (the type species of the genus Silicibacter Petursdottir and Kristjansson 1999) to the genus Ruegeria Uchino et al. 1999 as Ruegeria lacuscaerulensis (Petursdottir and Kristjansson 1999) Yi et al. 2007, comb. nov. According to Rule 37a, bacteriologists adhering to this proposal must change the name Silicibacter Petursdottir and Kristjansson 1999 to Ruegeria Uchino et al. 1999. 